\title{
[postprint version]
}

\section{Parenting an infant with a congenital anomaly: An exploratory study on patterns of adjustment from diagnosis to six months post-birth}

\section{Ana Fonseca, Bárbara Nazaré, \& Maria Cristina Canavarro}

\begin{abstract}
The present study examined psychological adjustment in parents of infants with congenital anomalies, focusing on the interval from the disclosure of the diagnosis to six months after the infant's birth, and considering the effects of the parent's gender and the timing of diagnosis (pre- vs. postnatal). Within-group diversity was also examined, by identifying distinct patterns of individual adjustment over time. Parents of 43 infants (43 mothers and 36 fathers) with a pre- or postnatal diagnosis of a congenital anomaly answered questionnaires assessing psychological distress and quality of life, one month after the disclosure of the diagnosis and six months after the infant's birth.

Results showed a significant reduction in psychological distress and a significant increase in Physical quality of life over time, for both parents, regardless of the timing of diagnosis. Fifty-seven per cent of parents presented a pattern of recovery from diagnosis to six months post-birth, and $26.6 \%$ presented a pattern of resilience. However, $15.2 \%$ of parents showed chronic adjustment difficulties. Findings suggest that most parents tend to adjust to their infant's CA, although some experienced difficulties and should be targeted for specialised counselling. Both members of the couple should be acknowledged, as both experience similar patterns of adjustment.
\end{abstract}


Key-words: parents of infants with a congenital anomaly, patterns of adjustment, psychological distress, quality of life.

\section{Introduction}

Congenital anomalies (CA) are structural or functional anomalies present at birth that arise during intrauterine development (Crowley, 2010). CA may occur in different body structures or systems, such as digestive system anomalies, nervous system anomalies, congenital heart disease, cleft lip and palate, among others (European Surveillance of Congenital Anomalies [EUROCAT], 2009). A pre- or postnatal diagnosis of a CA disrupts parental expectations of a healthy baby (Aite et al., 2003). Research has shown that the disclosure of a diagnosis of a $\mathrm{CA}$ is a distressing experience for parents, who display higher levels of psychological distress than parents of healthy infants (Fonseca, Nazaré, \& Canavarro, 2012; Kaasen et al., 2010). Furthermore, parents must adapt to the medical demands of the CA (e.g., surgeries, hospitalisations, increased medical monitoring) and to its associated challenges of care during the first months after their infant's birth (Mazer et al., 2008; Messias, Gilliss, Sparacino, Tong, \& Foote, 1995).

Existing qualitative studies have shown that parents experience a progressive reduction of their intense negative emotional reactivity, although some do experience continuing psychosocial difficulties over time (e.g., Aite et al., 2006; Drotar, Baskiewicz, Irvin, Kennell, \& Klaus, 1975). However, effective changes in parental 
adjustment over time can only be examined with prospective quantitative studies, which have been rare. The present study aimed to prospectively examine the adjustment of parents of infants with a $\mathrm{CA}$, from the moment of the diagnosis to six months after the infant's birth. Parents' individual adjustment was operationalized considering not only the presence/absence of psychological distress, but also the individual's overall wellbeing, i.e., individual's perception of quality of life (QoL; the subjective perception of well-being in the physical, psychological, social, and environmental life domains; The WHOQOL Group, 1994).

To our knowledge, only one study has examined parents' psychological distress from the diagnosis of their infant's CA to six months post-birth; that study found no significant changes between the two assessment points when the diagnosis occurred in the prenatal period, but a significant decline in psychological distress was found for parents whose infant's diagnosis occurred in the postnatal period (Brosig, Whitstone, Frommelt, Frisbee, \& Leuthner, 2007). Also, Skari et al. (2006) conducted a prospective longitudinal study assessing psychological distress few days, six weeks and six months post-birth in parents of infants with CAs. Although changes in parents' psychological distress over time were not examined, these authors showed that higher psychological distress levels few days after the infant's birth predicted higher psychological distress levels six months post-birth (Skari et al., 2006), suggesting some continuity in adjustment. Both studies found that parents in the prenatal group displayed higher 
psychological distress six months post-birth, compared to the postnatal group (Brosig et al., 2007; Skari et al., 2006). The prenatal diagnosis may be a long-lasting stressor because those parents deal with greater uncertainty until the infant's birth, when more information about the CA is available (Brosig et al., 2007).

In addition, one study found that parents of infants with a CA presented similar levels of QoL six weeks and six months after the infant's birth (Mazer et al., 2008). Similarly, in another study, mothers of infants with a prenatal diagnosis of a CA exhibited the same levels of satisfaction with life, at the moment of diagnosis and six months post-birth (Dale et al., 2012). To our knowledge, no studies examined the effect of timing of diagnosis on parents' QoL over time, which was a focus of this study.

Gender differences in parents' adjustment were also investigated. Regarding psychological distress, while some studies found no gender differences from the diagnosis to six months post-birth (Brosig et al., 2007), others found that existing gender differences in the early post-diagnosis stage (mothers experienced higher psychological distress than fathers, e.g., Fonseca et al., 2012) tended to be less pronounced six months post-birth (e.g., mothers experienced higher levels of anxiety, but not depression, than fathers, Skari et al., 2006) or even to disappear (e.g., no gender differences found three, six, nine, and 12 months post-birth, Pinelli et al., 2008), suggesting similarities between maternal and paternal experiences. When considering QoL, studies found that mothers presented lower QoL than fathers in the early post- 
diagnosis stage (Fonseca et al., 2012), but also six weeks and six months post-birth (Mazer et al., 2008).

Some studies support within-group diversity when parenting an infant with a CA, showing that while most parents present a gradual adjustment, some present chronic difficulties (e.g., 35.2\% of parents showed clinically significant psychological distress six months post-birth, Brosig et al., 2007). However, to our knowledge, individual patterns of adjustment over time have not been investigated. Research on individual adjustment to other stressful life events has identified four prototypical trajectories representing the heterogeneity of individual adjustment over time (Bonanno, 2004). The two most common trajectories are recovery (intense distress reactions in the acute phase followed by a gradual return to baseline functioning) and resiliency (maintenance of healthy adjustment over time, without disruption of functioning). In addition, a small proportion of individuals present chronically high levels of distress (chronic), while others display moderate distress levels in the acute phase, which progressively worsen over time (delayed; Bonanno, 2004; Bonanno, 2005; deRoonCassini, Mancini, Rusch, \& Bonanno, 2010).

The present study aimed to examine both parents' adjustment when parenting an infant with a CA, from the early post-diagnosis stage to six months post-birth, considering both group and individual (patterns of adjustment) levels. The effects of gender and timing of diagnosis were also examined. Parents' adjustment was 
operationalized as a multidimensional construct (Jomeen, 2004) that is not restricted to the absence of psychological distress, but also includes the subjective perception of physical, psychological, and social QoL. Although psychological distress and QoL are moderately related constructs - such that individuals with higher levels of psychological distress are more likely to present lower levels of QoL -, they do not overlap (Gameiro, Carona, Silva, \& Canavarro, 2010). Given our understanding of the literature, we hypothesise that: H1) parents will present lower psychological distress and similar levels of QoL over time; H2) parents in the prenatal diagnosis group will present higher psychological distress at T1 and at T2 than parents in the postnatal diagnosis group; $\mathrm{H} 3$ ) mothers will present lower QoL and higher anxiety symptoms than fathers over time.

\section{Methods}

Participants and Procedure

This study was approved by the Ethics Committees of the Hospitais da Universidade de Coimbra and the Centro Hospitalar de Coimbra (Portugal). Inclusion criteria for the study were: having an infant who was pre- or postnatally diagnosed with a CA, being at least 18 years of age, and having a level of literacy that permitted comprehension of the assessment protocol.

Data collection took place between September 2009 and February 2012. All parents who met the inclusion criteria were informed about this study by their medical 
team in a medical appointment, approximately one month after the disclosure of the diagnosis. The researchers presented the research goals to parents and those who decided to participate in the study signed an informed consent form, were given the questionnaires (Time $1-\mathrm{T} 1$ ) and were asked to return them to the researchers during the next medical appointment. Six months after the infant's birth (Time $2-\mathrm{T} 2$ ) the parents were mailed the questionnaires along with a pre-stamped envelope in which to return them after completion.

From the 82 couples initially contacted, 22 couples refused to participate or did not return the questionnaires at $\mathrm{T} 1$ (participation rate: 73.2\%). At T2, 17 couples did not return the questionnaires (attrition rate: 28.3\%). Participants who answered both assessments did not differ from the participants who dropped-out from the study, regarding socio-demographic characteristics and type of CA (data not shown). The final sample comprised 43 couples (43 mothers and 36 fathers). The sample characteristics are presented in Table 1. At T2, the infant's average age was 6.65 months $(S D=0.87)$.

[Insert_Table_1_about_here]

\section{Measures}

Psychological distress was evaluated with the Portuguese version of the Brief Symptom Inventory 18 (Derogatis, 2000), a 5-point Likert scale from 0 (Not at all) to 4 (Extremely), composed of three dimensions: Anxiety and Depression, which were used 
in the study, and Somatisation. Higher values indicate more intense psychopathological symptoms. Cronbach's alphas in our sample ranged from .88 (Depression -mothers) to .91 (Anxiety -mothers) at T1 and .92 (Depression -mothers) to .95 (Anxiety -fathers) at $\mathrm{T} 2$.

QoL was assessed with the Portuguese version of the World Health Organisation Quality of Life brief instrument (WHOQOL-Bref; Vaz-Serra et al., 2006), which consists of 26 items (answered on a 5-point Likert scale) organised into a facet of Overall QoL and four specific domains: Physical, Psychological, Social Relationships, and Environment. Higher scores indicate better QoL. In our study, the Overall facet of QoL and the Environmental domain were not used because they presented low Cronbach's alphas $(<.60)$ at one assessment time or more. In the remaining dimensions, Cronbach's alphas ranged from .61 (Social Relationships -mothers) to .82 (Psychological -fathers) at T1 and .61 (Physical -fathers) to .89 (Physical -mothers) at $\mathrm{T} 2$.

Sociodemographic (gender, age, marital status, educational level, and professional status) and clinical information (parity and history of pregnancy loss; the infant's data, including gender, gestational age at birth, type of CA, timing of diagnosis, hospitalization, and need for surgery) were collected.

\section{Data Analyses}


Analyses were conducted with IBM SPSS, version 19.0. Descriptive statistics were used to describe sample characteristics and parental adjustment. Chi-squared tests and $t$-tests were used to compare maternal and paternal sociodemographic characteristics.

To assess the effects of time, timing of diagnosis, and gender in psychological distress and QoL, repeated-measures MANOVAs were used, followed by univariate ANOVAs when the multivariate effect was significant. Gender differences were examined as a within-subjects factor within the couple.

To identify patterns of individual adjustment over time, parents were classified at both assessment times according to their scores on individual adjustment, using agglomerative hierarchical cluster analyses. The cluster variables (psychological distress and QoL) were standardised to Z-scores to equalise the contribution of each variable in the cluster analysis. Squared Euclidean distance was used to measure the distance between individual observations on the clustering variables and Ward's method of minimum variance was used to form the clusters. The number of clusters at each assessment time was selected based on the rescaled distances evident in the hierarchical cluster dendograms and the percentage change in agglomeration coefficients at each step of the cluster analysis (Hair \& Black, 2000). MANOVAs were performed to characterize the clusters and the frequency of parents in each cluster was reported. Continuity and change over time in cluster membership was examined using 
McNemar's test. Chi-squared tests were used to examine differences in individual patterns of adjustment over time as a function of gender and timing of diagnosis.

Significance was defined as $p<.05$, but marginally significant $(p<.10)$ effects are also reported. Post-hoc power calculations for the comparison analyses performed with a significance level of .10 and power $\geq .80$ indicated that large effects $(f \geq .33)$ could be detected (Faul, Erdfelder, Lang, \& Buchner, 2007). Effect-size measures are

presented for all comparison analyses (small: $\eta^{2} \geq .01$; medium: $\eta^{2} \geq .06$; large: $\eta^{2}=$ $.14)$.

\section{Results}

Parental adjustment: Effects of time, timing of diagnosis, and gender

Table 2 presents multivariate and significant univariate analyses of the main and interaction effects of time, timing of diagnosis, and gender. Figure 1 presents mean scores for parents' psychological distress and QoL at T1 and T2.

(Insert_Table_2_about_here)

Time. A marginally significant multivariate main effect of time was found for psychological distress and a significant effect was found for QoL. Univariate tests 
showed a significant decrease in Anxiety and Depression and a significant increase in Physical QoL (see Table 2 and Figure 1).

\section{(Insert_Figure_1_about_here)}

Timing of diagnosis. No significant multivariate main effects or interaction effects with time were found for psychological distress or QoL (see Table 2). Parents showed similar levels of adjustment and a similar pattern of change from $\mathrm{T} 1$ to $\mathrm{T} 2$, regardless of timing of diagnosis.

The timing of diagnosis $\mathrm{x}$ gender interaction was not significant for psychological distress, but it was significant for QoL, specifically in Social Relationships QoL (see Table 2). Post-hoc analyses showed gender differences at T1 for parents in the postnatal diagnosis group, with mothers $(M=81.67, S D=14.84)$ presenting higher Social Relationships QoL than their partners $(M=72.78, S D=17.10$; $Z=-2.31, p=.021)$. No gender differences were found in the prenatal diagnosis group or in either group at $\mathrm{T} 2$.

Gender. The multivariate main effect of gender was significant for psychological distress. Mothers presented higher Anxiety and Depression than fathers over time (see Table 2). Gender comparisons at each time-point showed that at $\mathrm{T} 1$ mothers presented higher Anxiety $\left(F=4.37, p=.044, \eta^{2}=.11\right)$ and Depression $(F=$ 
4.81, $\left.p=.035, \eta^{2}=.12\right)$ than fathers, although at T2 they presented higher Anxiety $(F=$ 5.61, $\left.p=.024, \eta^{2}=.14\right)$, but not Depression $\left(F=2.77, p=.105, \eta^{2}=.07\right)$, than fathers. The multivariate main gender effect for QoL was also significant, with mothers presenting lower Physical and Psychological QoL than fathers (see Table 2). Specifically, mothers presented lower Physical QoL than fathers at T1 $(F=7.05, p=$ $\left..012, \eta^{2}=.17\right)$ and lower Psychological QoL than fathers at T2 $\left(F=5.82, p=.021, \eta^{2}=\right.$ .14). Finally, none of the multivariate gender $\mathrm{x}$ time interactions was significant (see Table 2).

Individual patterns of adjustment: Continuity and change over time

A two-cluster solution provided the best fit for the data at $\mathrm{T} 1$ and $\mathrm{T} 2$. Participants belonging to Cluster 1 had significantly higher levels of psychological distress (T1: Pillai's Trace $=.26, F_{2,73}=12.95, p<.001, \eta^{2}=.26$; T2: Pillai's Trace $=$ $\left..73, F_{2,76}=101.70, p<.001, \eta^{2}=.73\right)$ and significantly lower QoL (T1: Pillai's Trace $=$ $.58, F_{3,72}=33.59, p<.001, \eta^{2}=.58 ;$ T2: Pillai's Trace $=.26, F_{3,75}=8.60, p<.001, \eta^{2}=$ .26) than participants in Cluster 2. According to their characteristics, Cluster 1 was labelled "Worse adjustment" and Cluster 2 - "Better adjustment".

(Insert_Table_3_about_here) 
Table 3 presents the cluster membership of the parents at each time-point. At T1, most parents were assigned to the "Worse adjustment" cluster, whereas most of the parents were assigned to the "Better adjustment" cluster at T2. The majority of participants changed cluster membership between T1 and T2 (McNemar's test for mothers: $p<.001$; fathers: $p<.001)$. In fact, the majority $(57.0 \%)$ of parents presented a pattern of recovery, and only one parent showed more distress at T2 than T1. With regard to continuity, $26.6 \%$ of parents presented a pattern of good adjustment over time (resiliency) and $15.2 \%$ of parents showed a pattern of chronic distress (see Table 3 ). No significant differences were found in the frequencies of the different adjustment patterns over time as a function of timing of diagnosis $\left(\chi_{3}^{2}=1.32, p=.725\right)$ or gender $\left(\chi_{3}^{2}=\right.$ $5.70, p=.127)$.

\section{Discussion}

The present study showed that, despite the initial impact of the diagnosis, most parents of an infant with a CA adapt to the condition, as shown by the improvement in their adjustment six months after the infant's birth. From the early post-diagnosis stage to six months post-birth, both mothers and fathers experienced a significant reduction in depression and anxiety symptoms, as well as a significant increase in Physical QoL (e.g., more energy, less fatigue, higher working capacity), supporting our first hypothesis. Similar results were found when considering individual patterns of 
adjustment: most of the parents showed a pattern of recovery over time. After the initial disruption following the disclosure of the diagnosis, parents usually seek more information about the CA and possible treatment plans (Aite et al., 2006), which reduces uncertainty and consequently improves parents' adjustment (Lipinski et al., 2006). Moreover, the parents' ability to readjust their expectations about the future, and the positive feelings associated with the infant's birth and parent-child interaction may alleviate the impact of the caregiving demands associated with the CA on parents' adjustment. Furthermore, $26.6 \%$ of parents were found to present a resilient pattern, that is the ability to maintain a good adjustment over time. These parents may be able to activate the internal and external resources needed to deal with their infant's CA, perceiving the situation as less disrupting and demanding (Boss, 2002). However, approximately $15 \%$ of parents presented chronic adjustment difficulties; they may have more difficulty in activating the resources to deal with the situation, or perceive it as very demanding (Boss, 2002). Moreover, there may be prior vulnerability factors compromising those parents' ability to adapt to their infant's CA. Future studies should explore which characteristics (e.g., individual characteristics, such as personal resources and perceptions) discriminate parents presenting different patterns of adjustment over time, to identify those who may present more adjustment difficulties as early as possible. 
Our results did not confirm our second hypothesis, suggesting instead that the process of parental adjustment to their infant's CA is similar regardless of the timing of diagnosis. Although parents whose infant has been prenatally diagnosed with a CA may experience uncertainty until the infant's birth (Brosig et al., 2007), they may also perceive this period as a time to prepare themselves to meet the infant's specific needs after birth (Nusbaum et al., 2008). Further studies should explore this hypothesis.

Our third hypothesis was partially confirmed. Our results showed that mothers presented more adjustment difficulties than fathers over time, but the individual adjustment trajectories were similar. Specifically, mothers presented higher Anxiety and Depression and lower Physical QoL than fathers at the early post-diagnosis stage, suggesting a greater impact of the diagnosis (Fonseca et al., 2012). Nevertheless, these gender differences also occur in the general population - due to physical changes resulting from the pregnancy and childbirth processes, and to the greater centrality of the parental role in mothers' lives (e.g., Jomeen, 2004; Woollett \& Parr, 1997) - and therefore may not be exclusively related to the impact of the diagnosis. However, mothers reported higher Social Relationships QoL than their partners only when the diagnosis occurred postnatally. One possible explanation is that in the post-birth period, parents tend to become closer to their nuclear family (Bost et al., 2002) and more distant from their extended social network; therefore, mothers (who usually benefit from maternity leave) may feel more protected from communication difficulties associated 
with the CA when the diagnosis is postnatal than when it is prenatal, leading to a greater perception of well-being in this life domain.

At six months post-birth, mothers still exhibited more adjustment difficulties than fathers (higher Anxiety and lower Psychological QoL) related to their role as primary caregivers (Hunfeld, Tempels, Passchier, Hazebroek, \& Tibboel, 1999). However, those differences tended to attenuate over time (no differences were found in Depression and Physical QoL). Mothers may have recovered from the physical changes of pregnancy and childbirth and may have managed to adapt to the new routines of caring for their infant, who also becomes increasingly less dependent on the exclusive maternal care; this may lead to a greater similarity between maternal and paternal experiences (Pelchat, Lefebvre, \& Perreault, 2003).

This study is an important contribution to the field for four reasons: a) it prospectively evaluates parental adjustment to an infant's CA; b) it considers psychological distress and QoL (a broader dimension of well-being) as adjustment indicators; c) it includes mothers and fathers; and d) it focuses on parents' adjustment to their infant's CA as a group, but also individually. However, this study also has limitations: a) the study's low power to detect small effects due to the sample size, and b) the non-categorical approach to CA (inclusion of different types of CA), which did not allow us to explore the influence of specific features and the severity of different CAs in parental adjustment. 
Finally, the findings of the present study lead to several clinical implications. First, health professionals should recognise the heterogeneity of individual patterns of parental adjustment to their infant's CA, and use that knowledge to anticipate parents' reactions and needs at different times. Although mothers presented more adjustment difficulties, especially at diagnosis, health professionals should be aware of the adjustment process of mothers and fathers, and assessment and intervention should include the participation of both parents whenever possible. A comprehensive parental assessment, focusing not only on their adjustment indicators but also on their appraisal of the situation and available resources (Boss, 2002), is essential to identify parents with a higher likelihood of developing chronic distress. Second, all parents may benefit from psychoeducation regarding the heterogeneity of individual trajectories in response to stressful events, which may help them normalise their reactions and self-identify indicators of adjustment difficulties. Finally, parents who present chronic adjustment difficulties should be targeted for specialised counselling. 


\section{References}

Aite L, Trucchi A, Nahom A, et al. (2003) Antenatal diagnosis of surgically correctable anomalies: Effects of repeated consultations on parental anxiety. Journal of Perinatology 23: 652-654.

Aite L, Zaccara A, Nahom A, et al. (2006) Mothers' adaptation to antenatal diagnosis of surgically correctable anomalies. Early Human Development 82: 649-653.

Aite L, Zaccara A, Trucchi A, et al. (2006) Parents' informational needs at the birth of a baby with a surgically correctable anomaly. Pediatric Surgery International 22: 267.270.

Bonanno G (2004) Loss, trauma, and human resilience: Have we underestimated the human capacity to thrive after extremely aversive events? American Psychologist 59: 20-28.

Bonanno G (2005) Resilience in the face of potential trauma. Current Directions in Psychological Science 14: 135-138. 
Boss P (2002) Family stress management: A contextual approach (2nd ed.). London: SAGE.

Bost K, Cox M, Burchinal M, et al. (2002) Structural and supportive changes in couples' family and friendship networks across the transition to parenthood. Journal of Marriage and Family 64(2): 517-531.

Brosig CL, Whitstone BN, Frommelt MA, et al. (2007) Psychological distress in parents of children with severe congenital heart disease: The impact of prenatal versus postnatal diagnosis. Journal of Perinatology 27: 687-692.

Crowley L (2010) An introduction to human disease: Pathology and pathophysiology correlations (8th ed.). Sudbury: Jones and Bartlett Publishers, LLC.

Dale M, Solberg O, Holmstrom H, et al. (2012). Mothers of infants with congenital heart defects: Well-being from pregnancy through the child's first six months. Quality of life research. 21 (1), 115-122Doi: 10.1007/s11136-011-9920-9.

Derogatis L R (2000). The Brief Symptom Inventory-18 (BSI-18): Administration, scoring and procedures manual. Minneapolis, MN: National Computer Systems.

deRoon-Cassini T, Mancini A, Rusch M, et al. (2010) Psychopathology and resilience following traumatic injury: A latent growth mixture model analysis. Rehabilitation Psychology 55(1): 1-11.

Drotar D, Baskiewicz A, Irvin N, et al. (1975) The adaptation of parents to the birth of an infant with a congenital malformation: A hypothetical model. Pediatrics, 56(5): 710-717.

European Surveillance of Congenital Anomalies [EUROCAT] (2009) EUROCAT guide 1.3 and reference documents: Instructions for the registration and surveillance of congenital anomalies. Newtonabby, County Antrim: EUROCAT Central Registry. 
Faul F, Erdfelder E, Lang AG, et al. (2007) G*Power3: A flexible statistical power analysis program for the social, behavioral, and biomedical sciences. Behavior Research Methods 39: 175-191.

Fonseca A, Nazaré B and Canavarro MC (2012) Parental psychological distress and quality of life after a prenatal or postnatal diagnosis of congenital anomaly: A controlled comparison study with parents of healthy infants. Disability and Health Journal 5: 67-74.

Gameiro S, Carona C, Silva S, et al. (2010). Qualidade de vida e depressão: Um estudo comparativo com doentes com diagnóstico clínico de depressão major, utentes de centros de saúde e indivíduos da população geral [Quality of life and depression: A comparative study with patients with major depression, patients from primary care units, and individuals from the general population]. In: Canavarro MC and Vaz Serra A (eds) Qualidade de vida e saúde: Uma abordagem na perspectiva da Organização Mundial de Saúde. Lisboa: Fundação Calouste Gulbenkian, pp. 299-324.

Hair JF and Black WC (2000) Cluster analysis. In: Grimm L and Yarnold PR (eds) Reading and understanding more multivariate statistics. Washington, D.C.: American Psychological Association, pp. 147-205.

Hunfeld J, Tempels A, Passchier J, et al. (1999) Parental burden and grief one year after the birth of a child with a congenital anomaly. Journal of Pediatric Psychology, 24(6): 515-520.

Jomeen J (2004) The importance of assessing psychological status during pregnancy, childbirth and the postnatal period as a multidimensional construct: A literature review. Clinical Effectiveness in Nursing 8: 143-155.

Kaasen A, Helbig A, Malt U, et al. (2010) Acute maternal social dysfunction, health perception and psychological distress after ultrasonographic detection of a fetal 
structural anomaly. British Journal of Obstetrics and Gynecology 17: 11271138.

Lipinski S, Lipinski M, Biesecker L, et al. (2006) Uncertainty and perceived personal control among parents of children with rare chromosome conditions: The role of genetic counseling. American Journal of Medical Genetics Part C (Seminars in Medical Genetics) 142C: 232-240.

Mazer P, Gischler SJ, Koot HM, et al. (2008) Impact of a Child with Congenital Anomalies on Parents (ICCAP) questionnaire: A psychometric analysis. Health and Quality of Life Outcomes 6(102): 102-110.

Messias D, Gilliss C, Sparacino P, et al. (1995) Stories of transition: Parents recall the diagnosis of congenital heart defect. Family Systems Medicine 13(3/4): 367-377.

Nusbaum R, Grubs R, Losee J, et al. (2008) A qualitative description of receiving a diagnosis of clefting in the prenatal or postnatal period. Journal of Genetic Counseling 17: 336-350.

Pelchat, D., Lefebvre, H., \& Perreault, M. (2003). Differences and similarities between mothers' and fathers' experiences of parenting a child with a disability. Journal of Child Health Care, 7(4), 231-247. doi: 10.1177/12674935030074001

Pinelli J, Saigal S, Wu YW, et al. (2008) Patterns of change in family functioning, resources, coping and parental depression in mothers and fathers of sick newborns over the first year of life. Journal of Neonatal Nursing 14: 156-165.

Skari H, Malt U, Bjornland K, et al. (2006) Prenatal diagnosis of congenital malformations and parental psychological distress: A prospective longitudinal cohort study. Prenatal Diagnosis 26: 1001-1009.

The WHOQOL Group (1994) Development of the WHOQOL: Rationale and current status. International Journal of Mental Health 23(3): 24-56.Vaz-Serra A, Canavarro MC, Simões MR, et al. (2006) Estudos psicométricos do instrumento de avaliação da qualidade de vida da Organização Mundial de Saúde 
(WHOQOL-Bref) para Português de Portugal [Psychometric studies of the World Health Organization Quality of Life assessment instrument (WHOQOLBref)]. Psiquiatria Clínica 27(1): 41-49.

Woollett A and Parr M (1997) Psychological tasks for women and men in the postpartum. Journal of Reproductive and Infant Psychology 15: 159-183. 
Anxiety symptoms

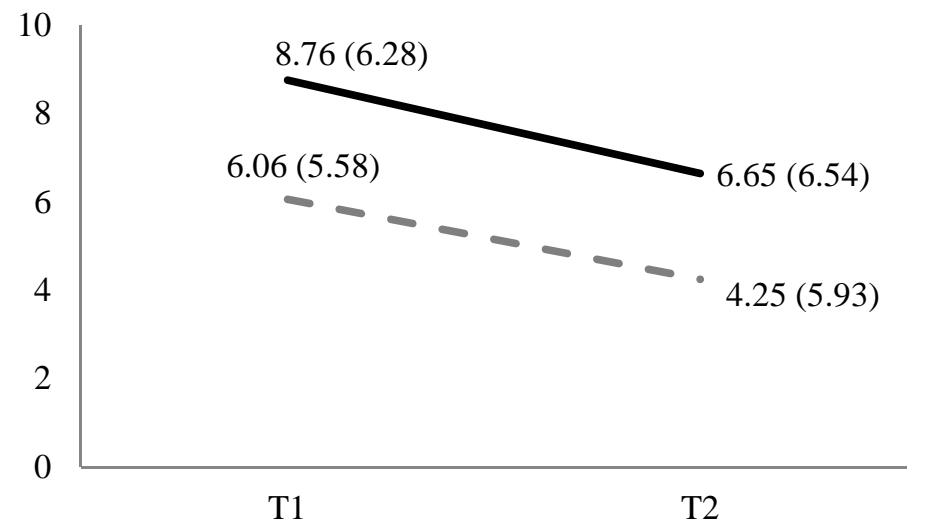

\section{Depressive symptoms}

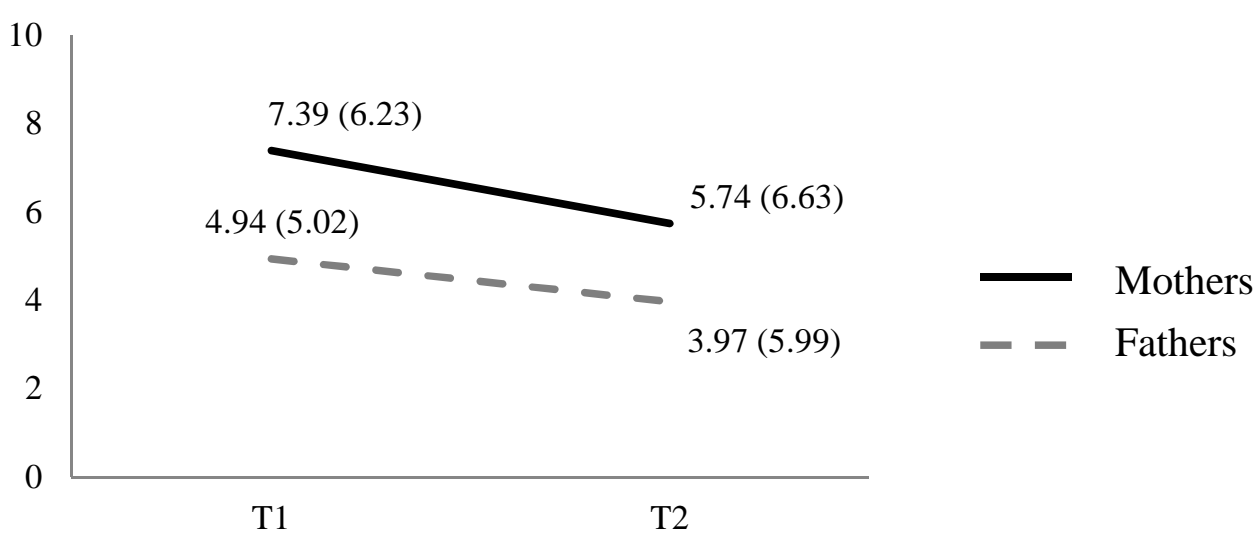

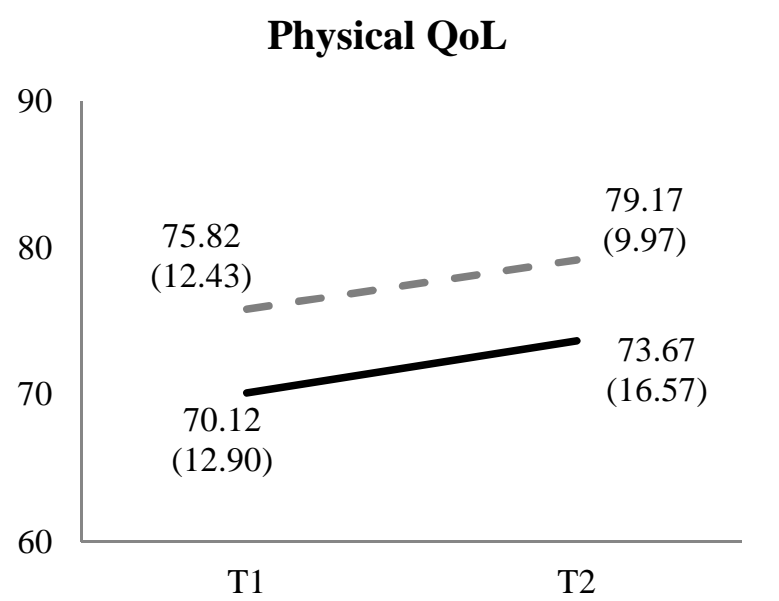
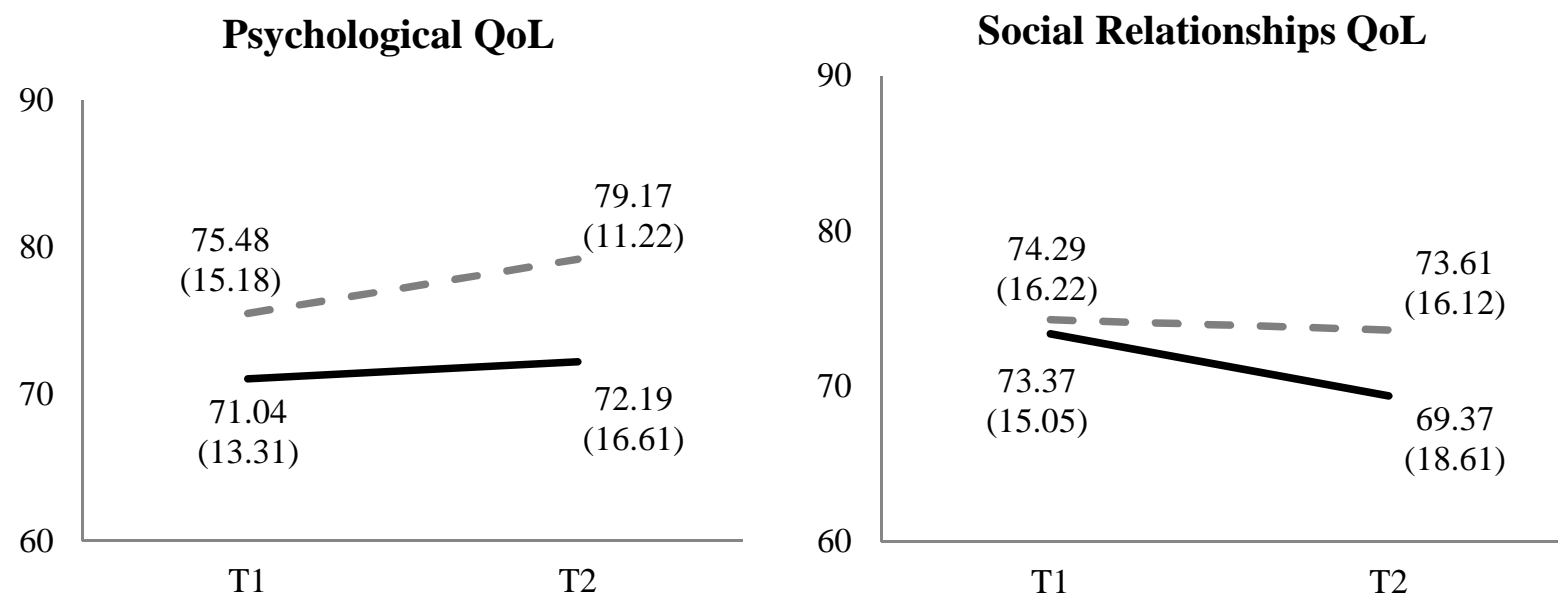

Figure 1. Parental psychological distress and quality of life at diagnosis (T1) and six months after the infant's birth (T2). 
Table 1 - Sample socio-demographic and clinical characteristics

$$
\text { Mothers }(n=43) \quad \text { Fathers }(n=36)
$$

Socio-demographic characteristics

\begin{tabular}{lccc}
\hline & $M(S D)$ & $M(S D)$ & $t$ \\
\hline Age & $31.58(4.95)$ & $33.25(5.05)$ & -1.48 \\
Educational level (years) & $14.07(3.53)$ & $12.11(2.73)$ & $2.69^{* *}$ \\
\hline \multicolumn{1}{r}{ Marital status } & $n(\%)$ & $n(\%)$ & $\chi^{2}$ \\
Married/Living together & $40(93.0)$ & $34(94.4)$ & 0.20 \\
Single/Divorced & $3(7.0)$ & $2(5.6)$ & \\
Erofessional status & & & \\
Enemployed & $8(18.6)$ & $33(91.7)$ & \\
\hline
\end{tabular}

Obstetric history

$$
\text { Mothers }(n=43)
$$

$$
n(\%)
$$

Parity

$\begin{array}{ll}\text { Primiparity } & 22(51.2) \\ \text { Multiparity } & 21(48.8)\end{array}$

History of pregnancy loss

$\begin{array}{ll}\text { Yes } & 8(18.6) \\ \text { No } & 35(81.4)\end{array}$

CA characteristics

Infant's data $(n=43)$ 
Prenatal

Postnatal

Type of congenital anomaly

Congenital heart disease

Nervous system anomalies

Digestive system anomalies

Urinary system anomalies

Oro-facial clefs

Limb anomalies

Hospitalization

Yes

No

Need for surgery

Yes

No
$13(30.2)$

$26(60.5)$

$17(39.5)$

$16(37.2)$

$5(11.6)$

$4(9.3)$

$11(25.6)$

$4(9.3)$

$3(7.0)$

$19(44.2)$

$24(55.8)$

$30(69.8)$

$p<.01$ 
Table 2 -Main and interaction effects of time, timing of diagnosis, and gender in psychological distress and quality of life: Multivariate and univariate effects

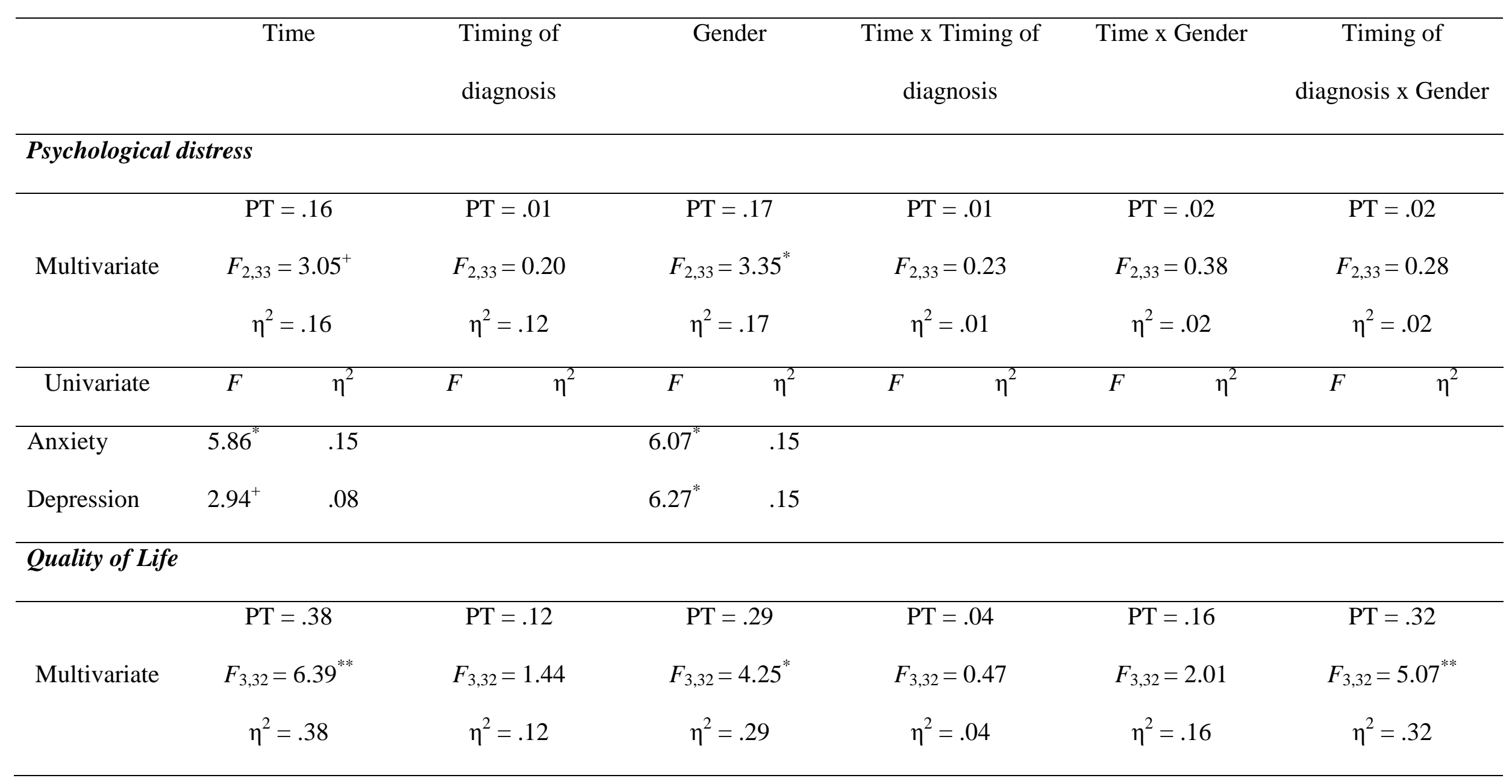




\begin{tabular}{|c|c|c|c|c|c|c|c|c|c|c|c|}
\hline Univariate & $F$ & $\eta^{2}$ & $F$ & $\eta^{2}$ & $F$ & $\eta^{2}$ & $F$ & $\eta^{2}$ & $F$ & $F$ & $\eta^{2}$ \\
\hline Soc. Relat. & 2.67 & .07 & & & 0.01 & .09 & & & & $4.76^{*}$ & .12 \\
\hline
\end{tabular}

Note. $\mathrm{PT}=$ Pillai's Trace.

${ }^{+} p<.10 .{ }^{*} p<.05 .{ }^{* *} p<.01$. 
Table 3 - Parents in each cluster of adjustment at diagnosis (T1) and six months after the infant's birth (T2).

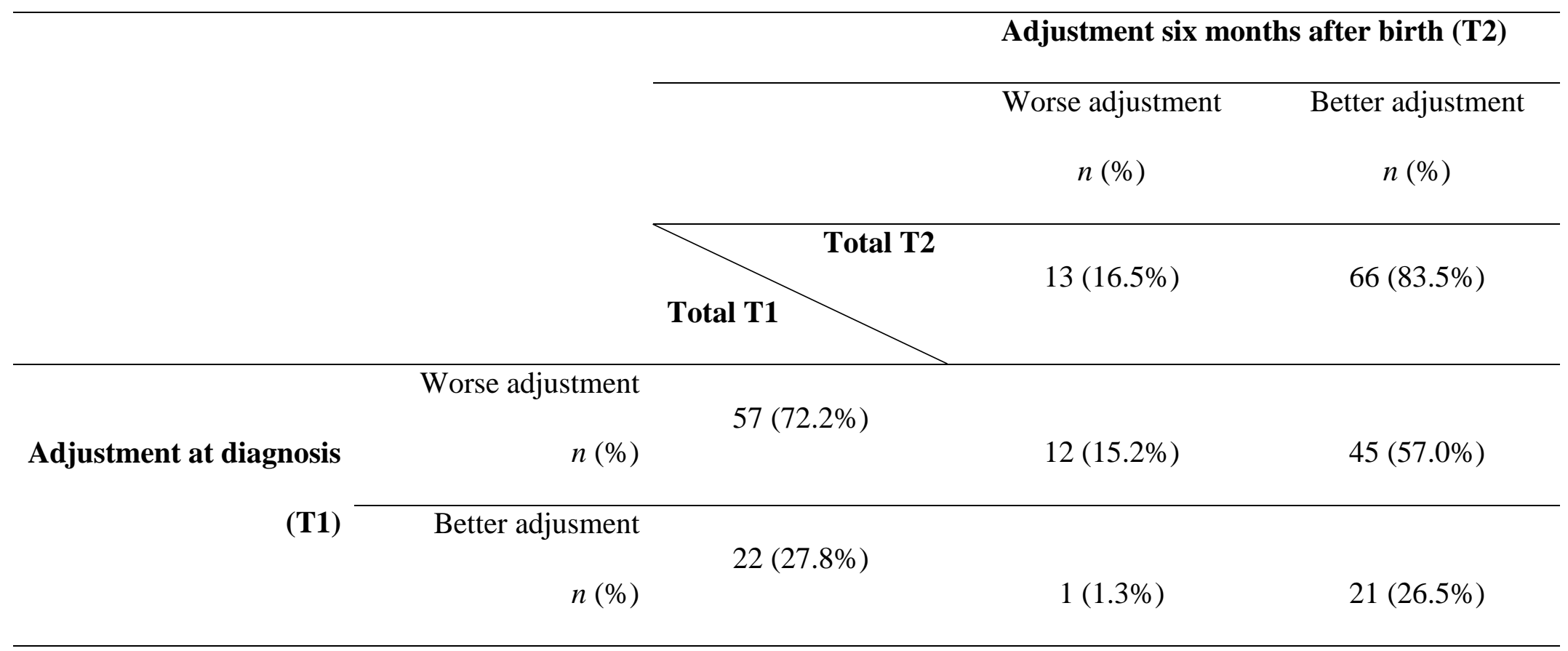

